Théologiques

Théologiques

\title{
Trois positions éthiques concomitantes
} En réaction à Geneviève Médevielle

\section{Melchior Mbonimpa}

Volume 14, numéro 1-2, automne 2006

Les lieux de la théologie aujourd’hui

URI : https://id.erudit.org/iderudit/014313ar

DOI : https://doi.org/10.7202/014313ar

Aller au sommaire du numéro

\section{Éditeur(s)}

Faculté de théologie et de sciences des religions, Université de Montréal

ISSN

1188-7109 (imprimé)

1492-1413 (numérique)

Découvrir la revue

Citer cet article

Mbonimpa, M. (2006). Trois positions éthiques concomitantes : en réaction à Geneviève Médevielle. Théologiques, 14(1-2), 107-114.

https://doi.org/10.7202/014313ar
Résumé de l'article

Cet article est une réaction à la contribution de Geneviève Médevielle qui porte sur la foi à l'oeuvre dans l'accueil des étrangers dans l'Église de France. Ce thème est abordé sous l'angle de l'éthique. N'étant pas moi-même éthicien, j'appuie mon commentaire sur une lecture longitudinale du texte, relevant au passage ce qui m'éclaire et m'enthousiasme, mais aussi ce qui soulève en moi des points d'interrogation. Ainsi, je remarque, entre autres choses, que les trois modèles d'éthique (éthique autonome, éthique de la foi, éthique communautarienne de la vertu) justifiant l'attitude des chrétiens qui entrent dans l'illégalité pour défendre des étrangers sans droits, ne devraient pas être présentés de façon diachronique. Il ne s'agit pas de positions concurrentes qui se suivent en se supprimant. Il faudrait plutôt les saisir comme des possibilités concomitantes et contemporaines.
Tous droits réservés ( Faculté de théologie et de sciences des religions, Université de Montréal, 2006
Ce document est protégé par la loi sur le droit d'auteur. L'utilisation des services d'Érudit (y compris la reproduction) est assujettie à sa politique d'utilisation que vous pouvez consulter en ligne.

https://apropos.erudit.org/fr/usagers/politique-dutilisation/ 


\title{
Trois positions éthiques concomitantes
}

\author{
En réaction à Geneviève Médevielle
}

\author{
Melchior MBONIMPA \\ Département de sciences religieuses \\ Université de Sudbury
}

Je voudrais d'abord féliciter ma collègue qui s'est acquittée de sa tâche avec rigueur et éclat, et je considère comme un honneur d'avoir été choisi pour réagir à son article. Je ne suis pas théologien moraliste, mais mon séjour dans le texte qu'on m'a fait parvenir en temps utile pour préparer cette réaction a été très instructif et enrichissant. Dès l'entrée, le titre: "La foi chrétienne, ressource pour vivre les questions de société » m'a accroché et intrigué, car ce qu'il annonçait ne va pas de soi : la foi n'a plus automatiquement droit de cité dans le contexte abordé par ce texte et les autres articles de ce numéro, c'est-à-dire l'Occident, qui se dit parfois "postchrétien ». Le croyant s'y trouve souvent comme en exil, mais l'exil, comme le présent, peut être vécu comme chance. Je reviendrai un peu plus loin sur cette belle expression que j'ai croisée à quelques reprises dans ma lecture de ce texte: "le présent comme chance». Mais avant cela, soulignons que le contenu de l'article est fidèle au titre: il nous révèle effectivement ce dont l'audace du recours à la foi est capable dans un contexte qui tente pourtant de marginaliser les croyants. Pour la suite de mes propos, je passerai à travers le texte, de manière longitudinale, relevant au passage ce qui me frappe, ce qui me rejoint, ce qui m'enthousiasme, ce qui m'éclaire, mais aussi ce qui soulève chez moi des points d'interrogation.

Dans l'introduction, j'ai été d'abord surpris qu'une brève note infrapaginale me fasse comprendre ce qu'est l'éthique procédurale. J'aurais dû être moins ignorant en ce domaine, parce que j'ai un ami qui a abordé cette question dans un certain nombre d'articles et d'ouvrages qu'il m'a systématiquement envoyés ${ }^{1}$. Au début, j'essayais de les lire, mais sans jamais

1. Il s'agit de Marc Maesschalck (voir 1994; 2001).

(C) Revue Théologiques 2006. Tout droit réservé. 
parvenir à me rendre très loin, parce que le recours au jargon spécialisé y était tellement intensif que j'avais l'impression de lire du sanscrit. Je justifiais alors ma démission en me disant que personne ne pourrait tout lire et tout comprendre et que la division du travail s'imposait. Maintenant, je sais que les textes sur l'éthique procédurale ne sont pas nécessairement hermétiques. Je ne me permettrais plus de mettre en cause trop vite le langage abscons des auteurs pour masquer la paresse intellectuelle du lecteur que je suis.

C'est aussi dans l'introduction que j'ai rencontré la formule à laquelle j'ai déjà fait allusion. On y parle d'une «Église qui, parce qu'elle interprète le présent comme chance, pense pouvoir contribuer au vouloir-vivre de notre société en faisant résonner les grandes affirmations de la foi : la valeur absolue de toute personne humaine, l'option radicale pour les pauvres, la quête de l'amour, du pardon et de la réconciliation au cœur de la violence, jusqu'au delà du mal». Il se pourrait que dans des sociétés relativement libres et prospères, l'interprétation du "présent comme chance " soit une excellente ruse pour permettre à la foi de se dire. Toutefois, dans d'autres contextes - qui ne sont pas du tout rares - (par exemple, au Rwanda et au Congo où des seigneurs de guerre se livrent à des massacres sans frein, ou encore au Nigeria où un islamisme importé désigne les chrétiens comme cibles uniquement parce qu'ils sont chrétiens), il me semble que le présent ne peut pas être interprété comme chance. L'Église n'a même pas besoin d'interpréter ce présent. Il lui suffirait de le regarder tel qu'il est, c'est-à-dire comme désastre, comme catastrophe ${ }^{2}$, pour être en mesure de répondre à la question éthique: que faire? C'est peut-être en prenant cet angle d'attaque que les chrétiens d'Afrique du Sud sont parvenus à la production d'un document consensuel, le «Document Kaïros ", qui, à sa manière, a contribué à accélérer la suppression de l'apartheid. Je note aussi dans l'introduction l'opposition entre «intransigeantisme et intégralisme». Un lecteur non spécialisé ou pressé pourrait ne pas saisir du premier coup que le terme « intégralisme » n'a pas la connotation négative que véhicule un autre terme de la même famille: «intégrisme».

Dans la seconde partie de l'exposé, il est question de « la foi à l'œuvre dans l'accueil de l'étranger dans l'Église de France». Nous y apprenons

2. Sur ce point, je partage plutôt le pessimisme de Walter Benjamin dans la onzième de ses "Thèses sur le concept d'histoire " où, commentant un tableau de Klee, il interprète le progrès comme une tempête qui emporte «l'ange de l'histoire " (représenté dans ce tableau) «irrésistiblement vers l'avenir auquel il tourne le dos, tandis que les décombres, devant lui, s'accumulent et montent jusqu'au ciel» $(2000,434)$. 
qu'il n'est pas exagéré de dire que la question de l'accueil de l'étranger est devenue "le symptôme des angoisses du pays en ce temps de crise ${ }^{3}$ ", et que l'accueil de l'étranger est vécu comme une épreuve. Cela m'a fait penser à un roman que j'ai lu récemment et qui m'a laissé perplexe. Le malaise qu'évoque la conférencière s'y trouve exprimé, mais de manière inversée. Le roman de Jean Raspail, Le camp des saints (1978), est un oracle de malheur qui prédit une gigantesque invasion de la France par des boat people venus de l'Inde, chargés d'un million d'immigrants, avant-garde du tiers-monde qui envahit l'Occident pour y trouver l'espérance. Le titre, comme on peut s'en rendre compte au fur et à mesure qu'on avance dans la lecture, est clairement polémique. "Les saints », ce sont ces damnés de la terre qui risquent de détruire l'Occident comme les barbares ont détruit Rome. Les saints, ce sont aussi les politiciens naïfs et pleutres qui refusent de voir, de prévoir, de prévenir le désastre, de soustraire l'Occident au péril barbare. Le roman est un plaidoyer pour la fermeture des frontières avant qu'il ne soit trop tard. Bien entendu, après la lecture d'un roman contenant un message aussi négatif, mais dans lequel une partie de la France se reconnaît plus que probablement, on ne peut qu'admirer le courage d'une Église qui se mobilise pour que l'étranger soit accueilli comme un frère: c'est une très bonne nouvelle!

On parle aussi du principe de laïcité dans cette partie. Je ne connais pas bien la généalogie de ce principe. Je me demande si les circonstances de sa naissance et ses conséquences concrètes dans l'histoire de la France, dans ses lois, dans ses politiques, pourraient expliquer pourquoi ce principe semble si contradictoire. D'un côté, la laïcité permet ou promeut le pluralisme. Elle est supposée rendre possible la coexistence pacifique de diverses croyances, religions ou convictions dans un même espace public. En ce sens, la laïcité se situe du côté de la tolérance. D’un autre côté, la laïcité française peut paradoxalement s'associer à l'intolérance, à la négation de la liberté. Un exemple récent de cette tendance est, bien évidemment, l'histoire du voile islamique, qui provoque bien des remous en France, mais pas tellement de ce côté-ci de l'Atlantique, même après le 11 septembre 2001. Et je me pose donc la question: le principe de laïcité fut-il à l'origine

3. Remarquons en passant que tous les temps sont «temps de crise » : toutes les époques, toutes les saisons sont saisies comme "temps de crise " par ceux et celles qui s'y trouvent impliqués et qui se heurtent à des "épreuves ". La seule différence entre notre temps et celui des autres (le passé, l'avenir ou un ailleurs spatial ou géographique) est qu'ici et maintenant, c'est nous qui faisons face à telle crise précise. 
et reste-t-il de nos jours une forme de neutralité positive destinée à favoriser la libre expression du pluralisme, ou, au contraire, une arme offensive dont le but est de réduire ou même d'éliminer l'influence de l'Église catholique, et plus tard, des autres confessions ou sectes qui tenteraient de recapturer les positions évacuées par les croyants?

Revenons maintenant sur le débat à propos de ce qu'il faut faire des étrangers. La conférencière nous dit: "Au regard de la loi qui veut qu'on reconduise aux frontières ces étrangers "sans-droits", certains chrétiens entrent dans l'illégalité et la "désobéissance civile» au nom de la fidélité à leur foi qui leur commande d'aimer Dieu et de le servir dans les plus petits des frères. » Je note en passant l'élégance de la formule «reconduire aux frontières " pour éviter la brutalité du terme "expulser ". Je trouve très belle l'attitude de ces chrétiens qui entrent dans l'illégalité pour défendre des étrangers sans droits, et je m'estime redevable à l'héroïsme de ces personnes engagées qui me permettent de revendiquer l'honneur d'être chrétien.

Dans cette même partie, je relève que ma culture générale s'enrichit en saisissant l'opposition entre l'éthique de responsabilité du gouvernement et l'éthique de conviction des chrétiens. Sur ce point, le texte se sert d'un cas et parle surtout des catholiques. Mais je serais curieux de savoir comment les protestants de France réagissent face au même problème. Et quels sont les alliés non chrétiens, peut-être même non croyants que le catholique trouve en première ligne, dans le même combat. Car, sur cette question précise de l'accueil de l'étranger, l'éthique de conviction, opposée à l'éthique de responsabilité, peut s'inspirer d'autres traditions spirituelles. Je pense notamment à l'impératif catégorique de la compassion dans le bouddhisme, mais aussi aux deux autres religions abrahamiques, le judaïsme et l'islam. L'une des qualités radicales que les trois monothéismes reconnaissent à Abraham, l'ancêtre-archétype, c'est l'hospitalité. La légende nous dit que la tente d'Abraham avait quatre portes toujours ouvertes vers les quatre points cardinaux, car l'étranger, émissaire possible du Dieu unique, pouvait venir de n'importe où et arriver n'importe quand.

À propos des catholiques de France, il faudrait peut-être se réjouir du fait qu'ils ne réagissent pas tous de la même manière face à la question de l'accueil des étrangers. Cela signifie qu'il n'y a pas de ligne de parti qui s'impose, et que ceux qui s'engagent ne sont pas contraints et forcés. C'est donc une heureuse absence d'unanimité. C'est aussi en ce sens qu'il faudrait apprécier le dilemme où se trouve l'Église de France, et qui est très bien exprimé dans l'alternative entre une défense des étrangers apparaissant 
comme une provocation politique et une abstention apparaissant comme un contre-témoignage. Peut-être qu'un tel dilemme empêche cette Église d'être trop sûre d'elle-même et d'oublier sa finitude et son humilité. Elle échappe ainsi à la tentation totalitaire d'une Église triomphante qui prescrit à tous l'autoroute vers la vérité tout entière.

Dans les deux dernières parties de l'exposé, j’ai de la difficulté à ne pas faire un choix parmi les trois modèles d'éthique abordés, c'est-à-dire l'éthique autonome, l'éthique de la foi, et l'éthique communautarienne de la vertu. En me situant dans le contexte occidental tel qu'il a été décrit dans le présent numéro, je ne parviens pas à m'empêcher d'avoir plus de sympathie pour l'éthique autonome que pour les deux autres. L'éthique autonome veut agir comme un levain dans la pâte du monde. Elle n'est pas démonstrative, mais je comprends que sa pertinence réside justement dans sa discrétion. Je ne saisis pas très bien l'objection qui dit que cette solution avait sa force dans une culture encore chrétienne. Cela contredit le fait que cette éthique est née justement de l'urgence de tenir compte d'un monde déjà sécularisé. Elle répond au besoin d'agir en tant que chrétien dans un monde déjà déchristianisé. Je ne voudrais pas contredire ce qui a été dit et redit dans les divers articles de ce numéro à propos du déclin de la théologie ou du christianisme occidentaux. Beaucoup d'intervenants nous ont présenté ce déclin comme récent, en fait, comme s'ils avaient été témoins de sa naissance. Mais d'après le peu que j'ai compris à l'école de ceux qui ont étudié la question de la déchristianisation (voir Delumeau 1977; Hervieu-Léger 1987; Raspail 1978), il me semble que le processus vient de très loin : de bien plus loin que Vatican II, sauf dans certaines enclaves comme le Québec d'avant la "révolution tranquille». Entre Maritain et nous, entre Vatican II et nous, s'est-il passé quelque chose qui ressemble à un changement de paradigme, comme le passage difficile du géocentrisme à l'héliocentrisme ? La difficulté réside peut-être dans la datation précise de la postmodernité qui rendrait caduque l'éthique autonome. Où est la ligne de partage entre la postmodernité et ce qui la précède ? Quel est l'événement ou l'ensemble des événements qui marquent ce passage ? Il me semble que les adversaires de l'éthique autonome renoncent à une nécessaire lucidité en voulant mettre fin à ce qu'ils appellent «l'aphasie de la foi » ou l'autosécularisation de l'Église. Ils renoncent à ce que Michel de Certeau désigne par une très belle expression « la faiblesse de croire » (1987), c'est-à-dire le courage d'admettre un fait têtu: que « la croyance est hors-laloi ». J'ai l'impression que la dénonciation de l'éthique autonome relève d'une fuite en avant. 
J'admets bien sûr la force du modèle de «l'éthique de la foi » et les prises de position qu'il rend possibles. J'aime certainement sa promotion du recours à la tradition. Je suis d'accord avec Simone Weil qui affirmait :

Il serait vain de se détourner du passé pour ne penser qu'à l'avenir. C'est une illusion dangereuse de croire qu'il y a même là une possibilité. L'opposition entre l'avenir et le passé est absurde. L'avenir ne nous apporte rien, ne nous donne rien; c'est nous qui, pour le construire, devons tout lui donner, lui donner notre vie même. Mais pour donner, il faut posséder, et nous ne possédons d'autre vie, d'autre sève, que les trésors hérités du passé et digérés, assimilés, recréés par nous. $(1949,50)$

Mais dans tous les dossiers, dans tous les défis où l'éthique de la foi exerce son droit de parole : euthanasie, clonage, avortement, etc., les chrétiens ne sont pas seuls: il y a des non-chrétiens qui défendent les mêmes positions en les justifiant autrement. Quels ponts, quelles passerelles ce modèle propose-t-il pour rejoindre les autres humains de bonne volonté et réaliser ainsi la seule forme de catholicité possible en ces temps-ci ? Par ailleurs, on peut bien sûr considérer qu'en elle-même, la prise de parole publique est déjà de l'ordre de l'action, mais est-elle plus efficace que la stratégie de l'éthique autonome au niveau des résultats ? Je pense qu' « en se prononçant massivement sur la vérité de la norme ", l'éthique de la foi favorise l'autoaffirmation aux dépens d'une collaboration avec des alliés possibles afin de défendre avec plus de succès les causes pour lesquelles les chrétiens se mobilisent.

C'est évident que l'éthique communautarienne de la vertu a quelque chose de très séduisant, de très rassurant et de très audacieux. Mais elle me fait penser aux Témoins de Jéhovah qui, tous les samedis matin, reviennent frapper à ma porte malgré le mauvais accueil que je leur réserve invariablement. Ce modèle me fait penser également à l'intrépidité des Esséniens qui se retiraient au désert pour prendre Yahvé à témoin de la corruption d'Israël, comme s'il pouvait y avoir un refuge hors du monde. J'ai l'impression que la stratégie de repli qu'adopte le «modèle sectarien » recèle une démission qui se cache sous les apparences du courage. Pour emprunter une image au bouddhisme, la cité vertueuse que visent ces "enclaves communautaires " ressemble à un "petit véhicule » qui, quand la maison commune est en train de brûler, sauve une toute petite minorité et abandonne le commun des mortels aux flammes.

En fait, il me semble que les trois modèles ne devraient pas être présentés de façon diachronique, comme s'ils se remplaçaient et se supprimaient 
successivement. Je serais plus à l'aise en les saisissant de façon synchronique, comme des possibilités concomitantes et contemporaines, car, dans ce cas, je me sentirais autorisé à exercer ma liberté de choix. Une présentation diachronique risque de suggérer qu'il n'y a qu'un choix, le dernier modèle, comme si le premier et le second étaient caducs. Tels sont les commentaires que m'ont inspirés le texte de notre collègue, la Professeure Médevielle, ainsi que, indirectement, les interventions de nos autres conférenciers invités et les réactions qu'elles ont suscitées.

\section{Références}

Benjamin, W. (2000), CEuvres III / trad. de l'allemand par M. de Gandillac, Paris, Gallimard (Folio. Essais 374).

Certeau, M. de (1987), La faiblesse de croire / texte établi et présenté par L. Giard, Paris, Seuil.

Delumeau, J. (1977), Le christianisme va-t-il mourir?, Paris, Hachettes.

Hervieu-Léger, D. (1987²) [1980], Vers un nouveau christianisme? Introduction à la sociologie du christianisme occidental, Paris, Cerf.

Maesschalck, M. (1994), Pour une éthique des convictions. Religion et rationalisation du monde vécu, Bruxelles, Publications des Facultés universitaires Saint-Louis.

- (2001), Normes et convictions, Zurich, Olms.

Morel, G. (1968), Problèmes actuels de religion, Paris, Aubier-Montaigne.

Raspail, J. (1978), Le camp des saints, Paris, Robert Laffont.

WeIL, S. (1949), L'enracinement. Prélude à une déclaration des devoirs envers l'être humain, Paris, Gallimard.

\section{Résumé}

Cet article est une réaction à la contribution de Geneviève Médevielle qui porte sur la foi à l'œuvre dans l'accueil des étrangers dans l'Église de France. Ce thème est abordé sous l'angle de l'éthique. N'étant pas moi-même éthicien, j'appuie mon commentaire sur une lecture longitudinale du texte, relevant au passage ce qui m'éclaire et m'enthousiasme, mais aussi ce qui soulève en moi des points d'interrogation. Ainsi, je remarque, entre autres choses, que les trois modèles d'éthique (éthique autonome, éthique de la foi, éthique communautarienne de la vertu) justifiant l'attitude des chrétiens qui entrent 
dans l'illégalité pour défendre des étrangers sans droits, ne devraient pas être présentés de façon diachronique. Il ne s'agit pas de positions concurrentes qui se suivent en se supprimant. Il faudrait plutôt les saisir comme des possibilités concomitantes et contemporaines.

\section{Abstract}

This article is in response to Geneviève Médevielle's contribution on the role of faith in welcoming foreigners by the Church of France. Her analysis focuses on the ethical dimension of the problem. As I am not an ethicist, my comments are based on aspects of the text which I find exciting or enlightening and others that raise questions for me. To cite an example, I find the three ethical models (autonomous ethics, faith ethics, community-based ethics of virtue), which justify the attitudes of Christians using illegal means to protect defenceless foreigners, should not be presented in a diachronistic fashion. It is not a question of successive competing positions which cancel each other out. The three positions are compatible and should be seen as concomitant possibilities. 